$$
\prod_{\substack{\text { UCRL-JC- } 117794 \\ \text { PREPRINT }}}
$$

\title{
Multiple Pulse Traveling Wave Excitation of Neon-like Germanium
}

\author{
J. C. Moreno, J. Nilsen, and L. B. Da Silva
}

This paper was prepared for submittal to the Conference Proceedings of 4th International Colloquium on X-ray Lasers

Williamsburg, VA

May 16-20, 1994

June 1994

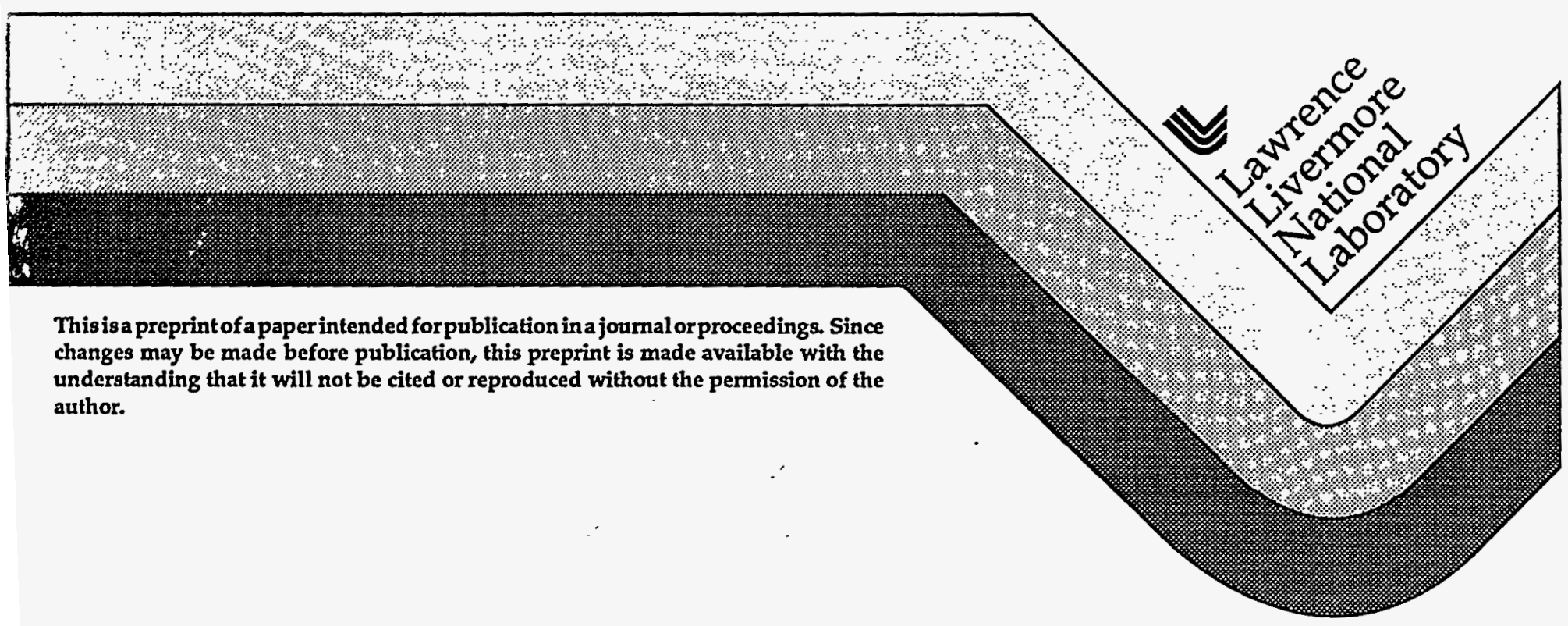
erstanding that it will not be cited or reproduced without the permission of the athor. 


\section{DISCLAIMER}

This report was prepared as an account of work sponsored by an agency of the United States Government. Neither the United States Government nor any agency thereof, nor any of their employees, make any warranty, express or implied, or assumes any legal liability or responsibility for the accuracy, completeness, or usefulness of any information, apparatus, product, or process disclosed, or represents that its use would not infringe privately owned rights. Reference herein to any specific commercial product, process, or service by trade name, trademark, manufacturer, or otherwise does not necessarily constitute or imply its endorsement, recommendation, or favoring by the United States Government or any agency thereof. The views and opinions of authors expressed herein do not necessarily state or reflect those of the United States Government or any agency thereof. 


\section{DISCLAIMER}

Portions of this document may be illegible in electronic image products. Images are produced from the best available original document. 


\title{
Multiple Pulse Traveling Wave Excitation of Neon-like Germanium
}

\author{
J. C. Moreno, J. Nilsen, and L. B. Da Silva \\ Lawrence Livermore National Laboratory \\ P. O. Box 808, Livermore, CA 94550
}

\begin{abstract}
Traveling wave excitation has been shown to significantly increase the output intensity of the neon-like germanium $x$-ray laser. The driving laser pulse consisted of three 100 ps Gaussian laser pulses separated by 400 ps. Traveling wave excitation was employed by tilting the wave front of the driving laser by 45 degrees to match the propagation speed of the $\mathrm{x}$-ray laser photons along the length of the target. We show results of experiments with the traveling wave, with no traveling wave, and against the traveling wave and comparisons to a numerical model. Gain was inferred from line intensity measurements at two lengths.
\end{abstract}

\section{INTRODUCTION}

Tilting the wave front of the driving laser to match the propagation velocity of the $x$-ray laser can be an effective method of increasing the effective gain length to extract the maximum energy out of the $x$-ray laser. This technique for exciting the plasma, usually called traveling wave excitation, is particularly useful and important when the duration of the driving laser divided by the length of gain medium is $\leq 33 \mathrm{ps} / \mathrm{cm}$ (the propagation time of $\mathrm{x}$-ray laser photons). It is expected that a table-top $\mathrm{x}$-ray laser will most likely require a short duration, high intensity driving laser to produce the plasma gain medium. A short duration $\mathrm{x}$-ray laser has many potential applications, such as probing a high density, laserproduced plasma or imaging a biological sample.

The traveling wave technique has been employed in various short pulse lasers to shorten the pulse duration and increase the amplification (1-5). Traveling wave excitation of an $\mathrm{x}$-ray laser has been demonstrated for the Ne-like yttrium exploding foil laser at $155 \AA$ (6). In these experiments we demonstrate the application of the traveling wave technique to the Ne-like Ge $x$-ray laser using slab targets. We observe a factor of ten increase in $x$-ray laser line intensity compared to no traveling wave and a factor of seventy increase compared to having the traveling wave go the opposite direction. Transit time effects are include in a numerical model that agrees well with the experiments. 


\section{EXPERIMENT AND DISCUSSION}

Experiments were performed using the Nova laser at Lawrence Livermore National Laboratory. The laser pulse consisted of three 100 ps Gaussian pulses separated by 400 ps peak to peak. Each pulse had an intensity on target of $1.1 \times$ $10^{14} \mathrm{~W} / \mathrm{cm}^{2}$ at a laser wavelength of $0.53 \mu \mathrm{m}$. A $3 \mathrm{~cm}$ long line focus of width $120 \mu \mathrm{m}$ was used. Targets consisted of a $1 \mu \mathrm{m}$ thick layer of Ge deposited on a $125 \mu \mathrm{m}$ thick foil of Ni. Because of a 16\% gap in the laser beam, the effective length of the target was $2.52 \mathrm{~cm}$. We also used half length targets for gain measurements. Our diagnostics consisted of a 1-meter grazing incidence spectrograph (McPigs) with a microchannel plate detector viewing one end of the $x$-ray laser axis and a flat field spectrograph (SFFS) using a varied line space grating and a streak camera detector viewing the opposite end of the $x$-ray laser.

The traveling wave was implemented by inserting a diffraction grating along with additional mirrors in the path of the driving laser (Nova), before the main amplifiers and KDP frequency doubling crystal. First order diffraction from the grating produces the tilted wave front due to a varying path length. The angle $\phi$ of the tilted wave front is given by $\tan (\phi)=\lambda \mathrm{L} / \mathrm{D} \cos \left(\vartheta_{0}\right)$, where $\lambda_{\mathrm{L}}$ is the laser wavelength $(1.06 \mu \mathrm{m}), \mathrm{D}$ is the groove spacing and $\vartheta_{0}$ is the diffraction angle. The grating groove spacing and incident angle $\vartheta_{i}$, was selected in this case so that the wave front was tilted to $\sim 45$ degrees to match the transit time of photons along the laser medium.

Germanium spectra taken from multiple pulse irradiated targets show a very strong $\mathrm{J}=0-1$ line unlike earlier experiments with a single pulse (7). In Fig. 1 we show a comparison between a multiple pulse spectrum and a single longer $(600$ ps) pulse spectrum. For the 600 ps Gaussian pulse experiment we observe the $\mathrm{J}=2-1$ lines at $232 \AA$ and $236 \AA$ but do not clearly see the $\mathrm{J}=0-1$ line. Other recent experiments of $\mathrm{Ne}$-like $\mathrm{Ge}$ with a slab target have also exhibited stronger lasing in the $\mathrm{J}=2-1$ lines $(8,9)$. Lasnex hydrocode simulations indicate that the multiple pulses are acting similar to the prepulse in that a larger more uniform density plasma is created compared to the standard single pulse (10). Streaked spectra of the plasmas show that the $\mathrm{x}$-ray laser lines do not show up during the first

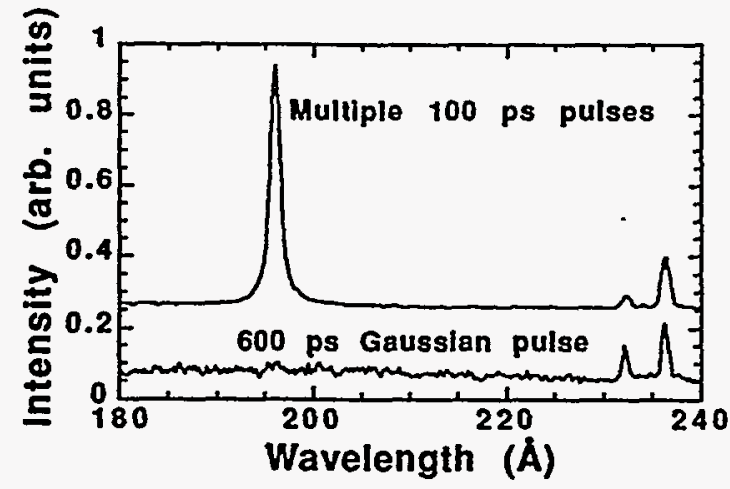

FIGURE 1. Comparison of Ne-like Ge x-ray laser spectrum for two pump laser conditions. pulse, but only appear during the second and third pulse of the driving laser. The first pulse ionizes the plasma but the conditions are unsuitable for gain. The second and third pulse create a 
somewhat hotter plasma with more gradual density gradients that are better suited for good amplification. The $\mathrm{J}=0-1$ line appears earlier in time than the $\mathrm{J}=2-1$ lines and has a somewhat shorter time duration.

We compared laser irradiation of Ge slab targets for three different wave front angles: first with the traveling wave going toward the detector $(\phi=45$ degrees); second with no traveling wave ( $\phi=0$ degrees); and third with the traveling wave going away from the detector ( $\phi=-45$ degrees). A simple numerical model was developed to simulate the effect of the transit time of the $x$-ray laser photons on the integrated output line intensity. In Fig. 2 we compare the measured integrated intensities of the $\mathrm{J}=0-1$ line at $196 \AA$, taken with the McPigs spectrograph, to this numerical model. The points show the measured data while the lines are from the numerical model. We measured a gain of $4.1 \mathrm{~cm}^{-1}$ for the $196 \AA$ line on data taken at two different lengths for the case with the traveling wave going toward the detector since there is no transit time effect for this case. For cases with no traveling wave or the traveling wave going in the opposite direction, there is a fall-off in the increase of intensity as the length of the plasma column is increased.

Our simulation assumed the time history of the emissivity and gain was Gaussian with a full-width at half-maximum (FWHM) of 85 ps for the $\mathrm{J}=0-1$ line at $196 \AA$. This emissivity time duration gave the best fit to our data for the $x$-ray laser going against the traveling wave. Experimental data in Fig. 2, for the case with no traveling wave, falls slightly below the expected values. This may be due to inhomogeneous

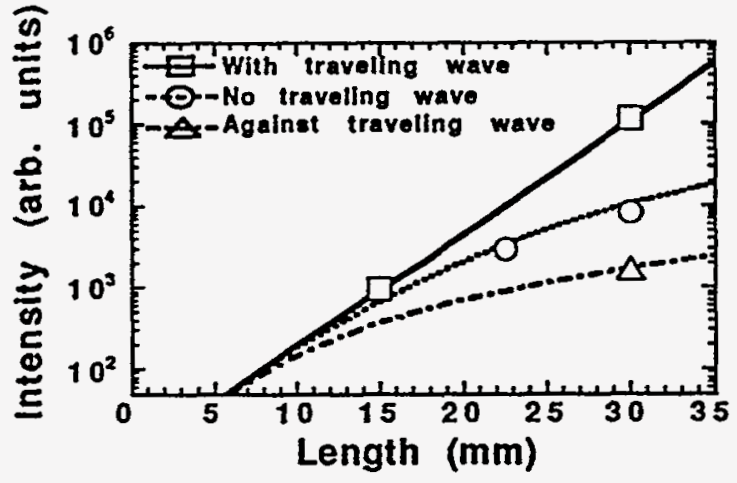

FIGURE 2. Intensity of the $\mathrm{J}=0-1$ line plotted vs length. The curves are from the numerical model while the points are experimental data. plasma effects and imperfect matching of the traveling wave pump to the propagation speed of the $x$-ray laser photons as well as approximations in the model. It should be noted that our model includes the $16 \%$ gap in the middle of the target since the propagation time across the gap is non-negligible.

The $\mathrm{J}=2-1$ line at $236 \AA$ was also measured for the three cases and exhibited a similar change in intensity due to the traveling wave. Again we adjusted the FWHM of the emissivity and gain time history in order to agree with the experimental data for the traveling wave going against the direction of the $x$-ray laser photon. In this case we have a somewhat longer duration (FWHM $=115 \mathrm{ps)}$ in our model consistent with the longer measured duration of the $236 \AA$ laser compared to the $196 \AA$ laser. The time duration of the $\mathrm{J}=2-1$ laser line at $236 \AA$ was measured for the three traveling wave configurations (at a plasma length of $2.52 \mathrm{~cm}$ ) and compared to the numerical model using the measured gain of 4.3 
$\mathrm{cm}^{-1}$. Our measurements give a laser duration (FWHM) of 55 and $77 \mathrm{ps}$ for the cases with and against the traveling wave as compared to model predictions of 36 and $64 \mathrm{ps,} \mathrm{respectively.} \mathrm{Agreement} \mathrm{with} \mathrm{our} \mathrm{model} \mathrm{is} \mathrm{reasonable} \mathrm{given} \mathrm{the}$ approximations in our model and the uncertainties in the plasma conditions.

\section{CONCLUSION}

We have compared $x$-ray laser experiments with, without and against the traveling wave. An order of magnitude enhancement of the Ne-like Ge x-ray laser intensity is observed when the traveling wave technique is used to compensate for the short gain duration of these plasmas. Gains of $4.1 \mathrm{~cm}^{-1}$ for the $196 \AA$ line and $4.3 \mathrm{~cm}^{-1}$ for the $236 \AA$ line were inferred from integrated line intensity measurements at two lengths. These measurements for the various traveling wave configurations and plasma lengths agree well with a numerical model including transit time effects. This technique can be important for applications of $\mathrm{x}$-ray lasers where a short pulse laser is required.

\section{ACKNOWLEDGMENTS}

The authors would like to thank S. Alvarez, T. Demiris, H. Louis, J. Ticehurst and the Nova operations crew for providing support for these experiments. This work was performed under the auspices of the U. S. Department of Energy by Lawrence Livermore National Laboratory under contract No. W-7405-ENG-48.

\section{REFERENCES}

1. R. Wyatt and E. E. Marinero, Appl. Phys. 25, 297-301 (1981).

2. Zs. Bor, S. Szatmari, and A. Muller, Appl. Phys. B 32, 101-104 (1983).

3. H. J. Polland, T. Elsaesser, A. Seilmeier, and W. Kaiser, Appl. Phys. B 32, 5357 (1983).

4. C. P. J. Barty et al, Phys. Rev. Lett. 61, 2201-2203 (1988).

5. M. H. Sher, J. J. Macklin, J. F. Young, and S. E. Harris, Opt. Lett. 12, 891-893 (1987).

6. L. B. Da Silva et al, "Imaging with $x$-ray lasers," Proceedings of SPIE, Ultrashort Wavelength Lasers II, 2012, 158-164 (1993).

7. J. Nilsen, J. C. Moreno, B. J. MacGowan, and J. A. Koch, Appl. Phys. B 57, 309-311 (1993).

8. T. N. Lee, E. A. McLean and R. C. Elton, Phys. Rev. Lett. 39, 1185 (1987).

9. D. Neely et al, Opt. Commun. 87, 231 (1992).

10. J. Nilsen, B. J. MacGowan, L. B. Da Silva, and J. C. Moreno, Phys. Rev. A 48, 4682-4685 (1993). 